\title{
EXPOSIMETRIC CHARACTERIZATION OF THE NEAR-FIELD OF A PORTABLE TRANSCEIVER EMITTING IN THE ULTRAHIGH FREQUENCY RANGE AND SIMULATION OF THE ELECTROMAGNETIC POWER DEPOSITED IN A FERRIMAGNETIC BIOLOGICAL TISSUE PRESENT IN SUCH A FIELD
}

\author{
Simona MICLĂUȘ*, Cora IFTODE**, Paul BECHET* \\ * "Nicolae Balcescu" Land Forces Academy, Sibiu, Romania \\ ** Politehnica University, Timișoara, Romania \\ simo.miclaus@gmail.com, cora.iftode@gmail.com, pbechet@gmail.com
}

\begin{abstract}
A portable radiocommunication device usually face-held during its use was chosen for characterizing its electromagnetic near-field distribution in air. Set to emit on a frequency of $440 \mathrm{MHz}$ for digital voice communication with a maximum input power of $5 \mathrm{~W}$, its antenna parameters were analyzed and the maps of field strengths were depicted up to distances of $20 \mathrm{~cm}$ from the device by using of a dual-sensor exposimeter. Since the occupational exposure safety limit for incident field levels was exceeded closer than $12 \mathrm{~cm}$ from the transceiver (for the magnetic field component), it became interesting to quantify the power loss in an alleged case of a brain containing also magnetite particles. Up to the present, only the electric field component was of interest when investigating biological effects of such exposures. With the new evidence from 2016, that human brain contains four orders of magnitude more magnetite nanocrystals than it was known before, a question rises in connection to the magnetic response of tissues impinged by fields with significant magnitudes and covering the hundreds of $\mathrm{MHz}$ frequency range. Starting from this question, we set-up a simulation in which a tissue with ferromagnetic content was mimicked for initial dosimetric computations.
\end{abstract}

Keywords: RF dosimetry, near field, head exposure, portable transceiver, biogenic magnetite

\section{Introduction}

Using portable transceivers for voice communication over distances of the order of kilometres needs antenna input powers of a few Watts. During its use the transceiver is positioned in front of the head, with the antenna at a few centimetres from eyes and brain. Following regulations that protect health against non-ionizing radiation, it is advised that incident field components - electric (E) and magnetic $(\mathrm{H})$ field strengths in air, to not exceed specified reference levels. Similarly, by using the specific absorption rate (SAR) of energy deposited in the passed tissue, a basic restriction for maximum admitted SAR in the head is provided. These limits are set for example in regulation [1] which is in use in Europe today, and they depend on the frequency (f) of the electromagnetic field (EMF).

A previous work of ours [2] highlighted that at distances shorter than 6-9 $\mathrm{cm}$ from a UV-B5 BaoFeng Amateur Portable Radio Transceiver emitting at $\mathrm{f}=446 \mathrm{MHz}$ a telephony signal modulated in frequency with a $12.5 \mathrm{kHz}$ channel bandwidth, the Eand $\mathrm{H}$-field strengths may exceed the 
reference levels even for occupational safety, when the input power in the antenna was $5 \mathrm{~W}$. Then, by evaluating SAR in liquids simulating the brain, based on Efield probe scans, we also identified overtaking of the basic restrictions for population, when the transceiver fed by 5 W was situated closer than $9 \mathrm{~cm}$ from the head phantom surface [2], [3].

Identifying a possible hazardous situation in this way, we were further interested to analyze the exposure to ultrahigh frequency (UHF) radiation in connection to a neglected aspect: magnetite nanoparticles, which are ferrimagnetic, are present in the brain and could absorb the $\mathrm{H}$-field component in a consistent manner [4]. Therefore we aimed to quantify the order of magnitude at which an UHF $\mathrm{H}$-field absorption takes place in ferrimagnetic fluids when they are exposed in the very proximity of a portable transceiver [5]. We have split the SAR evaluation problem in two: power loss assessment due to pure dielectric heating of tissues and power loss assessment due to magnetic heating of tissues when they contain ferro- or ferrimagnetic particles. Since presently the vast majority of SAR evaluations are based solely on E-field scans in simulating tissues, we raised the question if the $\mathrm{H}$-field component inside tissues like brain won't be also significant energetically or chemically from a biological point of view? With this background knowledge, current work synthetically points out the main outcomes of a prospective project dealing with UHF field absorption in tissues standing nearby communication devices.

\section{Objectives of the approach}

Present work had established three goals:

a. The experimental characterization of the monopole (whip) antenna model FA-S270C which is connected to the portable transceiver model ICOM ID 51 E plus, in the frequency band $430-440 \mathrm{MHz}$.

b. The mapping of the near-field around the transceiver, in air, separately on the two field components, $\mathrm{E}$ and $\mathrm{H}$, when it emitted voice signal on a carrier at $440 \mathrm{MHz}$ at an antenna input power of $5 \mathrm{~W}$.

c. Simulation of the near-field exposure given by the emission of the portable transceiver and determination of the power loss in a simple brain model containing few small spheres of magnetite and situated at a few centimetres from the device when it emitted a radiated power of $0.93 \mathrm{~W}$.

\section{Instruments, measurement procedure and simulation}

For the characterization of the antenna parameters, the FA-S270C monopole was connected to a ground plane and investigated inside a shielded and anechoic room (Figure 1). The measurements were made by using a vector network analyzer, a log-periodic receiver antenna and a special software. Three characteristics were determined: the return loss (S11), the antenna efficiency $(\eta)$ and the radiation pattern. For radiation pattern determination, fine rotations in both azimuth and elevation planes were enabled to the emitting antenna. The movements were conducted by an automated motor.

Two situations were studied: when the antenna was intact (with the rubber cover protecting its internal metallic structure present, like in usual use) and when the antenna was uncovered (just the internal metallic structure was present, without the rubber protection cover being applied).

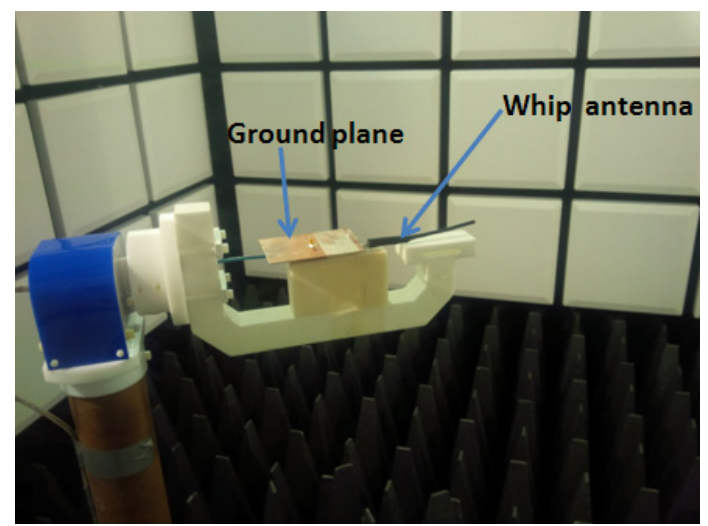

Figure 1: Experimental set-up for measuring the antenna parameters inside the anechoic room 
The measurements of the $\mathrm{E}$ - and $\mathrm{H}$-field strengths in air, around the transceiver, were made by the exposimeter ESM-30 RADMAN XT with data logging and RADMAN ESM-TS software, following a procedure described in [2]. Practically four vertical planes (parallel to the transceiver axis and to the antenna length) were scanned (in the face and in the back of the device, and in the two laterals of it).

The simulation of the wave propagation, of the power loss and SAR inside a ellipsoidal model of human brain containing five small spheres of magnetite was made in ANSYS HFSS software. The brain ellipsoid was positioned with its centre at a distance of 13 $\mathrm{cm}$ from the surface of the device and exposed in the near-field of the whip antenna radiating a power of $0.93 \mathrm{~W}$.

\section{Results and discussion}

Figure 2 depicts the variation with frequency of two antenna parameters: the reflexion coefficient, S11, and the efficiency of radiation, $\eta$. Uncovering the antenna doesn't modify the return loss S11, but the efficiency $\eta$ is highly dependent on the cover presence (it decreases due to the rubber envelope). This aspect is very important, showing that, from the total input power of $5 \mathrm{~W}$ in the antenna, depending on the cover state and its material, the effective isotropic radiated power (EIRP) will be more or less reduced. In consequence, the input power will not be a reference parameter when speaking about exposure, until exact measurements providing real EIRP values are made.

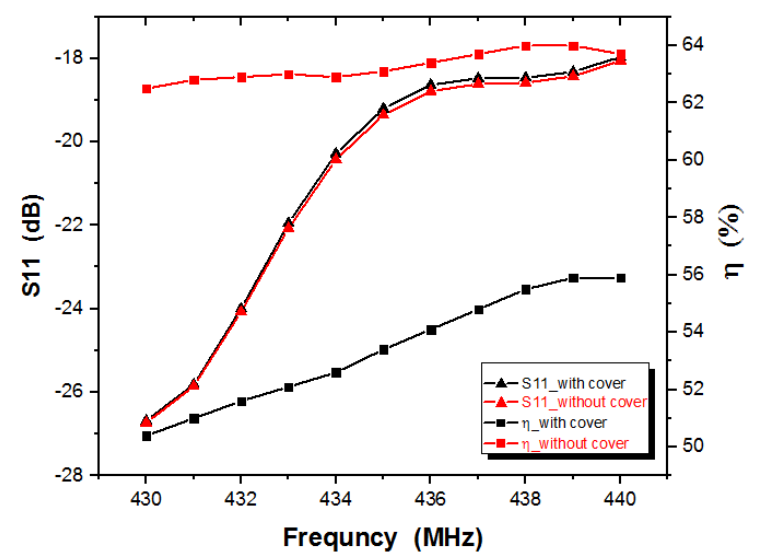

Figure 2: Return loss and efficiency of the covered versus uncovered antenna FA-S270C

Figure 3 presents the radiation pattern of the antenna when covered or when uncovered by the rubber envelope. At $\mathrm{f}=440 \mathrm{MHz}$, the total gain is in the order $0.27-0.37 \mathrm{dBi}$ and a good isotropy of the radiation is observed in the gain diagram. The presence of the cover on the antenna reduces the maximum gain with $0.1 \mathrm{~dB}$ and also the efficiency to radiate with $7.7 \%$. However, in the real use, the whip antenna is

covered.
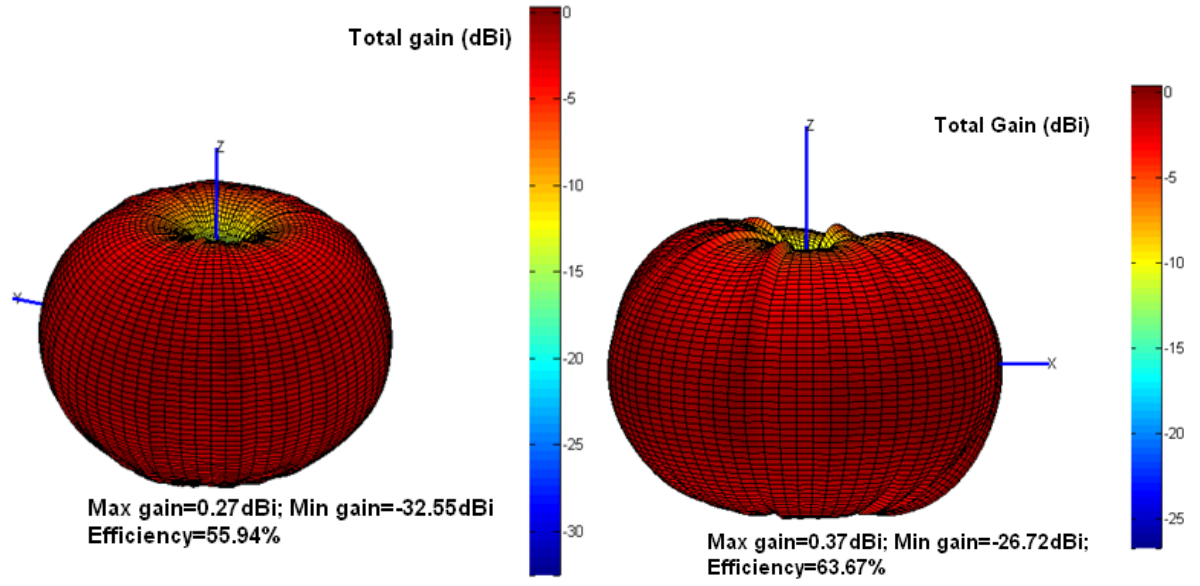

Figure 3: Radiation pattern of the antenna FA-S270: covered by the usual rubber envelope (left) and uncovered (right) 
Figure 4 shows the distribution of the rootmean-square (rms) values of the E-field strength in air in four vertical planes in the proximity of the transceiver. At $\mathrm{f}=440 \mathrm{MHz}$ the E-field is highest nearer to the antenna upper end. If compared with the reference levels given in [1], which indicates maximum admitted limits for safe use at this frequency in case of occupational exposure, E_limit $=62.9 \quad \mathrm{~V} / \mathrm{m}$ and $\mathrm{H}$ _limit $=16.7 \overline{\mathrm{A}} / \mathrm{m}$, the E-field strength in air is exceeded for distances shorter than 9 $\mathrm{cm}$ from the device. Given that the E-field exceeds safe limits in the upper part of the transceiver, where the brain usually is positioned, a possible hazard may appear if the device is held closer.

Figure 5 depicts the rms values of $\mathrm{H}$-field distribution in air in the vicinity of the ICOM transceiver. A larger volume around the device presents high values of $\mathrm{H}$-field level, than in the case of E-field level. For the $\mathrm{H}$-field strength safety limitation, it results that using the device at distances shorter than $12 \mathrm{~cm}$ from the head/face should be avoided when the input power of $5 \mathrm{~W}$ is used at $440 \mathrm{MHz}$ voice transmission. Both measurements of $\mathrm{E}$ - and $\mathrm{H}$-fields took place in a usual room, and one notes that a good symmetry of field distribution over 360 degrees around the transceiver was obtained.
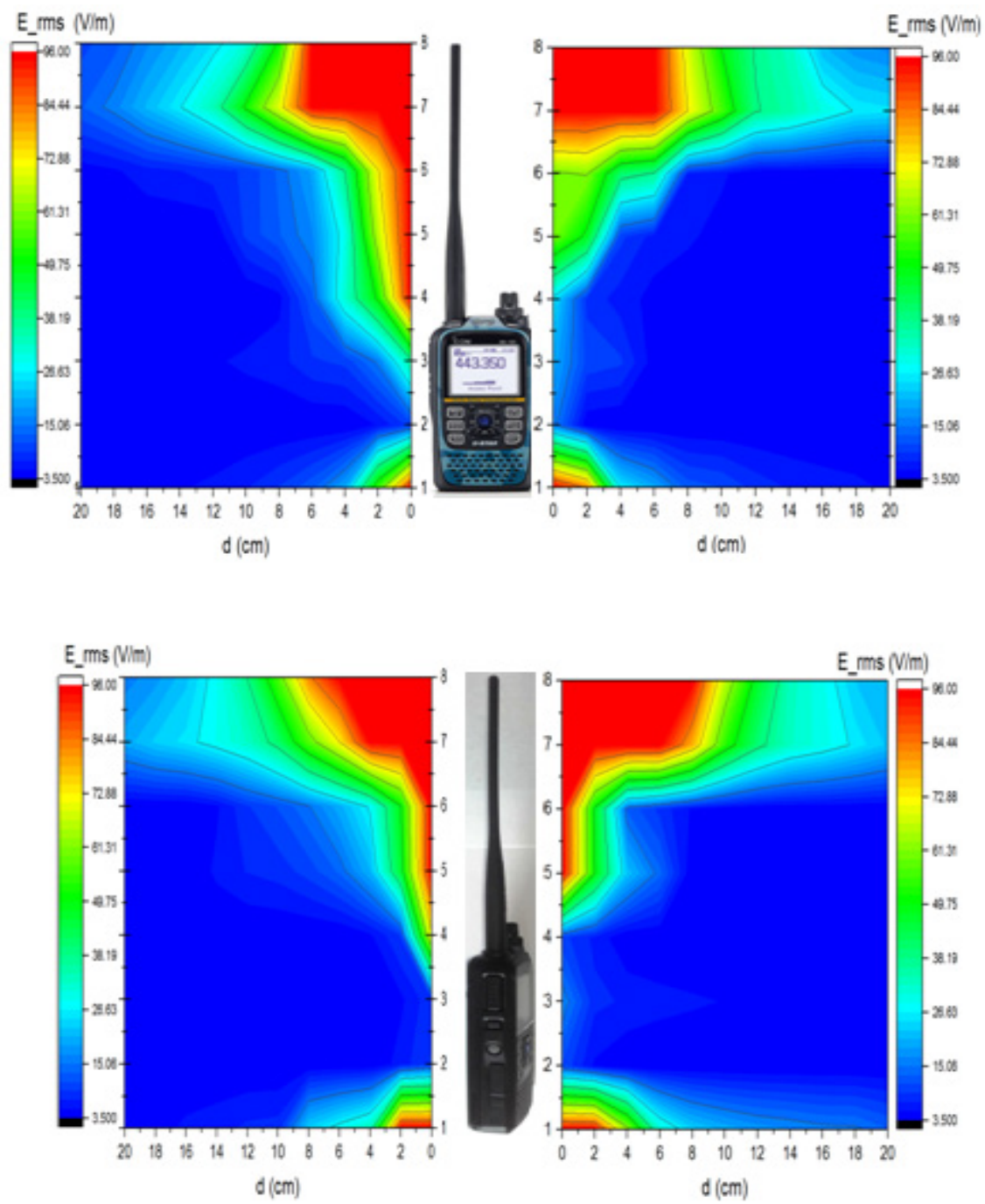

Figure 4: Map of electric field strength measured around the transceiver ICOM ID 51 E plus, in air, digital voice emission, $f=440 \mathrm{MHz}$, input power in the antenna of $5 \mathrm{~W}$

In Figure 6 it is emphasized the volume loss density distribution in the modelled 

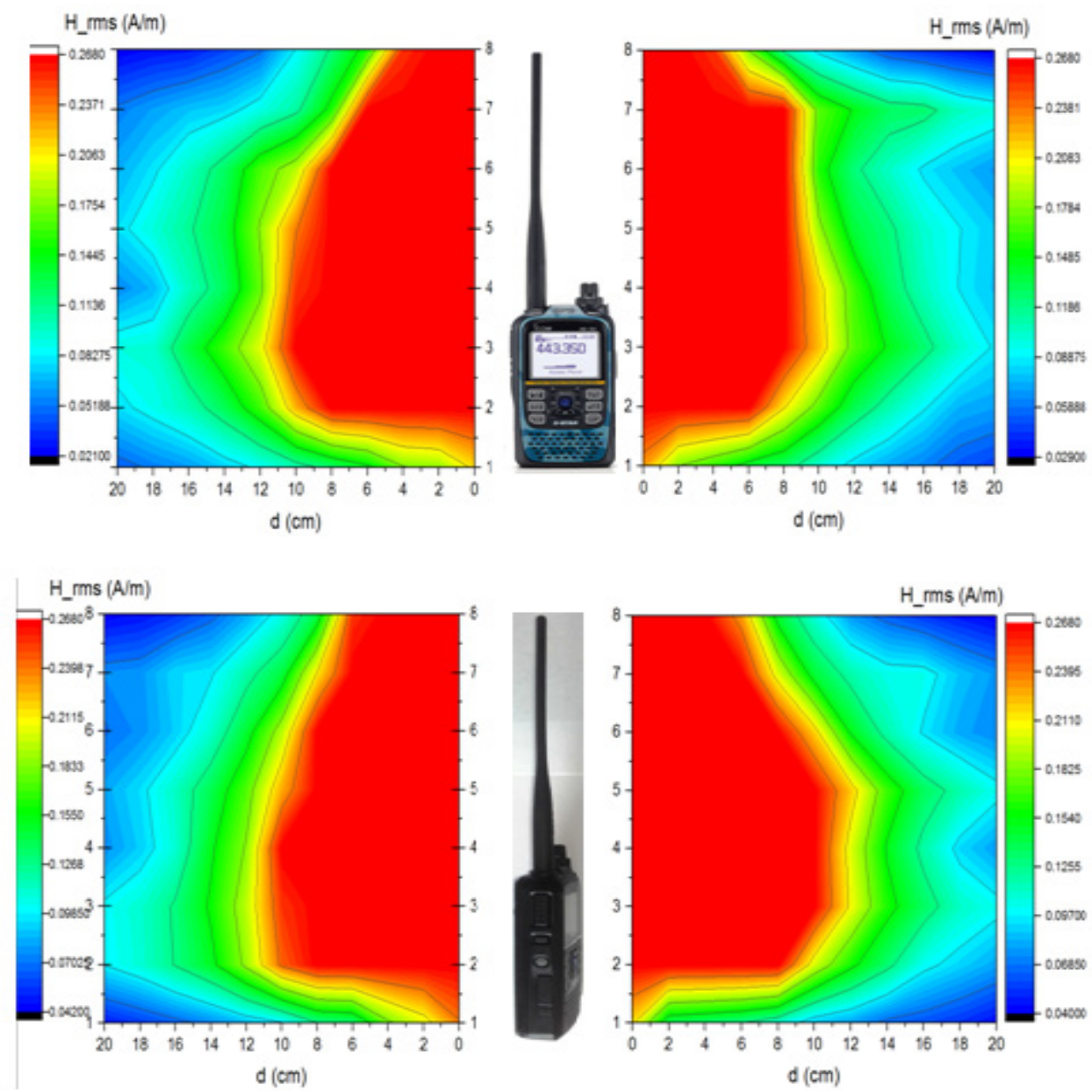

Figure 5: Map of magnetic field strength measured around the transceiver ICOM ID 51 E plus, in air, digital voice emission, $f=440 \mathrm{MHz}$, input power in the antenna of $5 \mathrm{~W}$

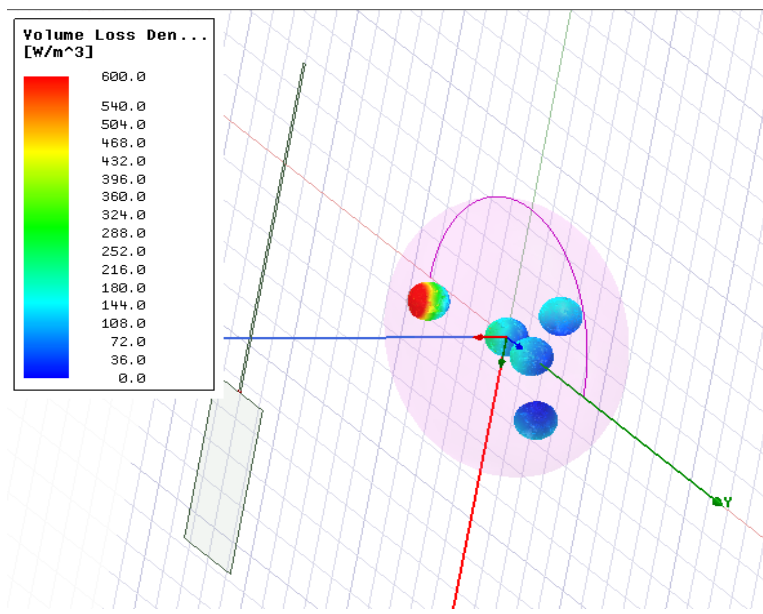

Figure 1: Volume loss density distribution in the five magnetite particles spread in the ellipsoidal model of dielectric brain centre at $13 \mathrm{~cm}$ from the antenna emitting at $440 \mathrm{MHz}$ and $0.93 \mathrm{~W}$ radiated power magnetite particles present in the ellipsoidal brain, taking into account the cumulative effect of $\mathrm{E}$ and $\mathrm{H}$ fields. If SAR would have been computed directly by the ANSYS HFSS software, just the E-field strength would have been counted for. The new method applied here is able to provide the cumulative $\mathrm{E}-$ and $\mathrm{H}$ field contribution to SAR.

For current calculations we used a brain model of volume $=1130 \mathrm{~cm}^{3}$ (and dielectric properties of human brain tissue) containing five spheres of magnetite (with electric and magnetic properties taken at $440 \mathrm{MHz}$ [5]) with radius $=1 \mathrm{~cm}$ and a monopole antenna with input power $=1 \mathrm{~W}$ and a computed $\eta=92.6 \%$. In this case we obtained a mean $\mathrm{SAR}=0.055 \mathrm{~W} / \mathrm{kg}$ in the brain, which is 
$3.1 \%$ less than the mean SAR computed based only on E-field absorption considerations. If translated in current experimental exposure case, where the input power was $5 \mathrm{~W}$ and measured $\eta=55.9$ $\%$, the current experimental average SAR would be of $0.166 \mathrm{~W} / \mathrm{kg}$. Of this, 5.1 $\mathrm{mW} / \mathrm{kg}$ are due solely to $\mathrm{H}$ field component dissipation in magnetite spheres.

To be noted in Figure 6 that the closest magnetites sphere to the antenna shows much higher power loss density than the distant ones. Therefore, even if this simple situation shows that average SAR value is far from exceeding the safety limits (of 2 $\mathrm{W} / \mathrm{kg}$ ), local power deposition in magnetite material may be much increased.

\section{Conclusions}

When human head is placed in front of a portable transceiver occupationally used for digital voice transmission at $440 \mathrm{MHz}$, and for an input power in the antenna of $5 \mathrm{~W}$, the safety for the incident electric field influence is ensured for distances larger than $9 \mathrm{~cm}$, and for magnetic influence - for distances larger than $12 \mathrm{~cm}$. Not the input power will represent the indicator of the possible hazard due to exposure, but the effective isotropic radiated power, which needs determination for each particular situation. The rubber cover protecting the antenna, its material and its state will also impact on the radiation efficiency and therefore on the field strength distribution around the device.

Checking the response of a simulated brain volume situated at the afore-mentioned distances scale from the monopole antenna of the transceiver, we could get the total power loss density. With the brain model in which small particles of magnetite were spread in order to mimic real brain properties (both electric and magnetic), we observed that even if average SAR values were far from alarming, a contribution of around $3 \%$ from total SAR could be acknowledged solely to magnetite contribution. Besides, it may happen that magnetite particles, at least at their surface, to become hot spots of power deposition. According to their position in the neural system, the magnetite particles may still pose problems, even when heating will not be significant, since their role in the brain is still unknown.

These findings sustain further research on this topic for providing answers to the safe use of such devices on the long term.

\section{References}

[1] International Commission on Non-Ionizing Radiation Protection (ICNIRP), Guidelines for limiting exposure to time varying electric, magnetic, and electromagnetic fields, Health Physics, Vol. 74, pp. 494-522, 1998.

[2] Miclaus S., Dumbrava I., Voicu V., Bechet P., Patru I., Electromagnetic exposure due to portable two-way radio transceivers and walkie-talkies, Proceedings of the $10^{\text {th }}$ International Symposium on Advanced Topics in Electrical Engineering, pp. 346-349, Bucharest, Romania, March, 2017.

[3] Miclaus S., Dumbrava I., Voicu V., Bechet P., Electromagnetic dosimetry of radiofrequency portable transceivers used in front of the face, Proceedings of the International Conference Knowledge-Based Organization, Vol. XXIII, No. 3, pp. 65-70, June 2017.

[4] Kirschvink J. L., Microwave absorption by magnetite: A possible mechanism for coupling nonthermal levels of radiation to biological systems, Bioelectromagnetics, Vol. 17, pp. 187-194, 1996.

[5] Miclaus S., Racuciu M., Bechet P., H-field contribution to the electromagnetic energy deposition in tissues similar to the brain but containing ferrimagnetic particles, during use of face-held radio transceivers, Progress in Electromagnetic Research B, Vol. 73, pp. 49-60, 2017. 